\title{
Mathematical model of the crystallizing blank's thermal state at the horizontal continuous casting machine
}

\author{
Igor Yu. Kryukov', Sergey M. Gorbatyuk², and Margarita G. Naumova ${ }^{2, *}$. \\ ${ }^{1}$ Thyssen Krupp AG Representative office Russian Federation, Russia, 105120, Moscow, 2 Siromyatnichesky proezd, 1 \\ ${ }^{2}$ National University of Science and Technology MISIS, Russia, 119049, Moscow, Leninsky prospect, 4
}

\begin{abstract}
Present article is devoted to the development of the mathematical model, which describes thermal state and crystallization process of the rectangular cross-section blank while continious process of extraction from a horysontal continious casting machine (HCCM). The developed model took cue for the heat-transfer properties of non-iron metal teeming; its temperature on entry to the casting mold; cooling conditions of blank in the carbon molds in the presence of a copper water cooler. Besides, has been considered the asymmetry of heat interchange from blank's head and drag at mold, coming out from fluid contraction and features of the horizontal casting mold. The developed mathematical model allows to determine alterations in crystallizing blank of the following factors with respect to time: temperature pattern of crystallizing blank under different technical working regimes of HCCM; boundaries of solid two-phase field and liquid two-phase filed; blank's thickness variation under shrinkage of the ingot's material
\end{abstract}

\section{Introduction}

The proposed mathematical model is meant for studying of the casting metal's thermal states the at the horizontal continuous casting machine (HCCM) of fine silver alloy $[1,2]$.

The casting mold of the investigated HCCM was made of graphite and is intended for casting of rectangular cross-section blanks $[3,4]$. A feature of its design is a presence of a copper cooling unit (Fig. 1). The basic geometry of the horizontal graphite mold is shown in Fig 2 and of the copper cooler in Fig. 3 [5]. The graphite casting mold and the copper cooler are used for production of the rectangular section blanks $200 \times 20 \mathrm{~mm}$.

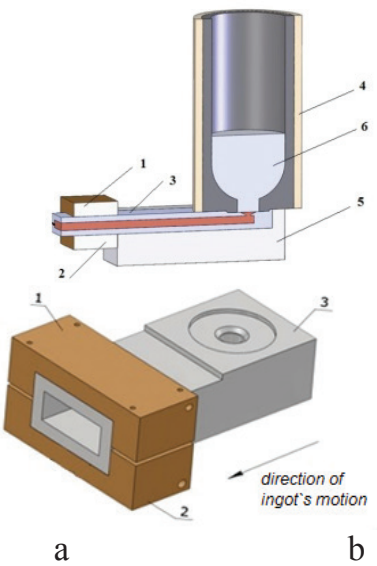

Fig. 1 General arrangement drawing of the HCCM for production of the rectangular section blanks (a) and graphite mold with copper cooler (b): 1 - the top part of the cooler; 2-the bottom part of the cooler; 3 - the top part of the graphite mold; 4crucible; 5- heat-insulating material; 6- heat- insulator.
In the scientific works dedicated to the investigations of the ingot's thermal state in the continuous-casting molds CCM $[6,7]$ pointed out, that in the liquid core of the cast slab the heat transfers as a result of combination of both heat conduction and convection. The reasons for this are natural convection of overheated melt, rabbling of the melt by force of supplied hot metal, electromagnetic effect and other exposures over the ingot's liquid core. The motion of the molten metal in the unhardened part of the ingot significantly complicates the calculation of the ingot's solidification, since towards the correct accounting of the heat- and mass transfer in the solution phase, should be taken into account not only the heat-transfer equation, but the system of equations of flows and continuity [6].

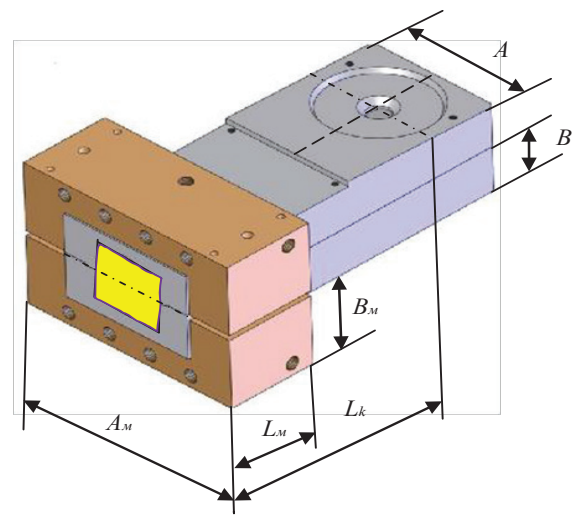

Fig. 2 Basic dimensions of the HCCM

\footnotetext{
* Corresponding author: qwerty-rita@yandex.ru
} 


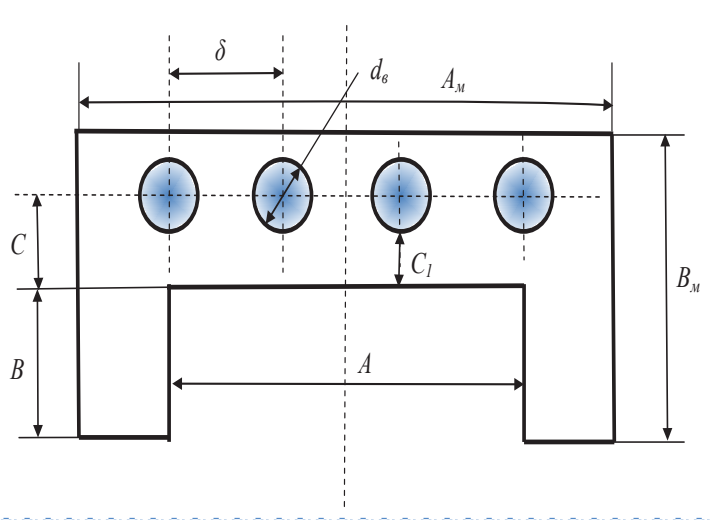

Fig. 3 Basic dimensions of the copper water cooler

The problem can be simplified by introducing an effective heat conductivity coefficient of the liquid phase $\lambda_{\text {ef, }}$ whose value is extended several times in comparison to the molecular thermal conductivity coefficient $\lambda_{1}$.

$$
\lambda_{\mathrm{ef}}=\varepsilon_{\mathrm{con}} \lambda_{\mathrm{l}}
$$

where $\varepsilon_{\text {con- }}$ - coefficient of convection, depending on the melt motion intensity. In view of low initial velocity of the metal stream flow rate in the HCCM, we possess the value $\varepsilon_{\text {con }}=1 \div 2$ [6]. Consequently, the effective heat conductivity of the liquid phase can outnumbers the conductivity of the stationary phase. The increasing of the effective heat conductivity in the hot metal while stirring, leads to a heat-transfer rise at the front of the phase transition, to the heating of the solid phase and to the associated heattransfer rise at the ingot's surface.

When steady-state operating conditions the concerned horizontal graphite mold HCCM has in different parts the temperature from $100^{\circ} \mathrm{C}$ to $600^{\circ} \mathrm{C}$. For this temperature range, for the thermal conductivity $\lambda_{t}$, has been obtained an equation of linear regression:

$$
\lambda(t)=127,28-0,11657 t, \mathrm{~W} /(\mathrm{m} \mathrm{K}),
$$

where $\lambda_{\mathrm{t}}$ - is the coefficient of conductivity of graphite in the direction perpendicular to extrusion.

With its application the mean integral value of conductivity in this temperature range is:

$$
\lambda_{\mathrm{gr}}=\bar{\lambda}=\frac{1}{600-100} \int_{100}^{600} \lambda(t) d t \approx 86,5 \mathrm{~W} /(\mathrm{m} \mathrm{K})
$$

It is well- known, that silver and its alloys are possible to cast at a temperature only 50 degrees above liquidus temperature. The casting speed, or the drawing speed generally depends on the blank's width, on the melt temperature on entering the casting mold and on properties of metal. The pacing factor, determining the casting speed is a thickness of a solid crust on exit from the casting mold. The water velocity in the canals generally depends on the width $a$ and the thickness $b$ of the blank and on the casting speed $v$ (Table 1).
Table 1. Critical process parameters of ingot's draw-forming from $\mathrm{HCCM}$

\begin{tabular}{|c|c|c|c|c|}
\hline № & Name title & Parameter & Unit & Value \\
\hline 1 & $\begin{array}{l}\text { Degree of su- } \\
\text { perheat of the } \\
\text { melt on the } \\
\text { exit from the } \\
\text { crucible }\end{array}$ & $\Delta T_{0}$ & ${ }^{\circ} \mathrm{C}$ & $50-100$ \\
\hline 2 & $\begin{array}{l}\text { Melt tempera- } \\
\text { ture on exit } \\
\text { from the } \\
\text { melting cruci- } \\
\text { ble }\end{array}$ & $T_{0}=T_{l}+\Delta T_{0}$ & ${ }^{\circ} \mathrm{C}$ & $920-1020$ \\
\hline 3 & Casting speed & $v$ & $\mathrm{sm} / \mathrm{min}$ & $10-20$ \\
\hline 4 & $\begin{array}{l}\text { Cooling water } \\
\text { temperature }\end{array}$ & $T_{w}$ & ${ }^{\circ} \mathrm{C}$ & 25 \\
\hline 5 & $\begin{array}{l}\text { Water veloc- } \\
\text { ity in canals }\end{array}$ & $W_{w}$ & $\mathrm{M} / \mathrm{s}$ & $1-4$ \\
\hline
\end{tabular}

\section{Basic assumptions and limitations of the mathematical model}

The scope of the blank's solidifying task is solved in the Cartesian coordinate system. For the origin of coordinates was taken a point of intersection of diagonals of rectangle, generated by the inner passage section of the casting mold, which is going through the crucible's axis. The orientation of the reference axes and the direction of the ingot's motion $\vec{v}$ is shown in Figure 4. It is apparent; that in general case the thermal process modeling area is a parallelepiped $A B C D A$ ' $B$ ' $C$ ' $D$ '.

When developing of mathematical model of the ingot's thermal state were adopted the following assumptions and limitations:

1. We assume, that the temperature in the given point of the ingot in stationary casting regime is a timeindependent quantity (in the Earth coordinate system), that is , we consider a quasipermanent thermal conditions;

2. We consider as the temperature significantly varies along the axes $x$ and $z$. The thing is, that the thickness of the ingot $b$ is much more less than its width $a$, the design of the mold and the cooler are such, that the cooling is more intensive on the side of faces $B B^{\prime} C^{\prime} C$ and $A A^{\prime} D^{\prime} D$ (Figures 3 and 4). The boundary conditions on the side face $A B C D$ are same as on $A^{\prime} B^{\prime} C^{\prime} D^{\prime}$, that is, the symmetry of the temperature fields with respect to the plane $E F G H$ is observed, providing $d T / d y=0$. To achieve the set modeling problem, for a sake of simplicity, we adobe the convention that $d T / d y<<d T / d x$. The modeling work area of the ingot's thermal state is the rectangle $E F G H$.

3. The quasi-equilibrium model is used to describe the process of liquid metal solidification, with there is a belief that, there is no diffusional supercoooling in the mushy zone;

4. In the cooling zone (where copper cooler is used), on its border with the graphite mold, should be taken the average value of the thermal transmission coefficient, which, in its turn, depends on the cooler design and on parameters of quenching water ; 
5. We assume that in the direction of axis $\mathrm{Oz}$ the heat is transferred only by convection. In witness of it, the Peclet number is the determining factor for the convection - heat conduction relationship:

$$
P e=\operatorname{Re} \cdot \operatorname{Pr}=\frac{W_{z} \Delta z}{a},
$$

Where $\Delta z$ - typical length scale along the motion, where temperature gradient $\mathrm{dT} / \mathrm{dz}$ is observed, this distance can be estimated as $L_{k} \approx 330 \mathrm{~mm}=0,33 \mathrm{~m} ; W_{z}=v$ - the casting speed.

The minimum casting speed $W_{z}=v=10 \mathrm{sm} / \mathrm{min}=16,6$ $10^{-4} \mathrm{~m} / \mathrm{s}$, the thermal conductivity coefficient for solid phase in the neighborhood of $T s$ is $a=1,2210^{-4} \mathrm{~m}^{2} / \mathrm{s}$. In these condition $P e \approx 5$. In fact, for $P e>1$ the heat propagation through the heat conduction can be neglected. It means, that if we choose two different points 1 and 2 , which have the same $x, y$ coordinates and different $z$ coordinate values $\left(z_{2}>z_{1}\right)$, then the temperature of the second point depends on the first point's temperature, and the temperature of the first point doesn 't depend on the second point's one for the selected direction of the ingot's motion (Figure 4).

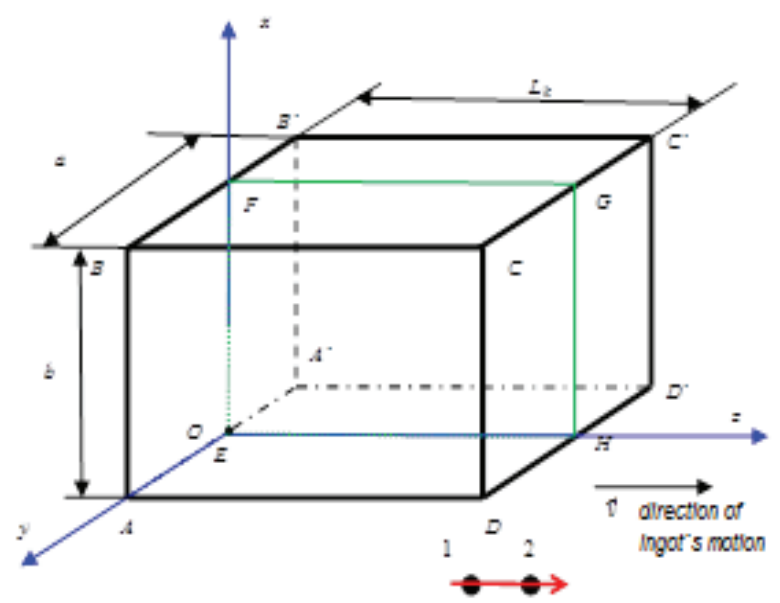

Fig. 4 Modeling area of ingot's thermal state and the selected cartesian reference system.

\section{Mathematical model of the ingot's thermal state}

The mathematical model of the ingot's thermal state is based on the nonlinear equation of the heat conduction, which is complemented with initial and boundary conditions; at the same time the solidification heat is accounted according to the quasi-equilibrium model of the twophase zone.

According to the first and the second suppositions, the temperature distribution in the solidifying ingot (in the frame reference associated with the Earth) can be described by the steady-state energy equation, taking into account the autothermal heat supply

$c \rho v \frac{\partial T}{\partial z}=\frac{\partial}{\partial x}\left(\lambda \cdot \frac{\partial T}{\partial x}\right)+\frac{\partial}{\partial z}\left(\lambda \frac{\partial T}{\partial z}\right)+q_{\mathrm{v}}(x, y, z, \tau)$
Let us distinguish the control volume $V_{0}$ in the twophase zone of the ingot and, in the same time, to introduct in the calculations the rate of the hard phase $\psi=V_{\text {sol }} / V_{0}$ ratio of the hard phase volume to the total volume of the distinguished element of the two-phase zone (hard phase proportion).In the liquid phase parameter $\psi$ is equal to zero $\left.\psi\right|_{T=T_{s}}=0$, for a solid part of the ingot it is equal to one ${ }_{\left.\psi\right|_{T=T_{s}}}=1$ and in the two phase zone it varies from zero to one; $\mathrm{T}_{\mathrm{s}}$ and $\mathrm{T}_{l}$ - consequently the solidus and the liquidus temperatures of the blank's material [6].

We will assume, that the heat source function $q_{v}(x, y$, $z, \tau)$ is directly-proportional to the local solidification rate

$$
q_{\mathrm{v}}(x, y, z, \tau)=\rho L \partial \psi / \partial \tau
$$

where heat of melting $L$ acts as a proportionality coefficient.

We consider the thermal state of an object in the coordinate system, associated with an arbitrary cross section of the blank, which is in motion with the ingot. Taking into account the ratio $d z / d t=v$, we get:

$$
c \rho \frac{\partial T}{\partial \tau}-\rho L \frac{\partial \psi}{\partial \tau}=\frac{\partial}{\partial x}\left(\lambda \frac{\partial T}{\partial x}\right)+\frac{\partial}{\partial z}\left(\lambda \frac{\partial T}{\partial z}\right)
$$

In the general case the solidification speed $d \psi / d t$ in the Eq.7 is determined by conformity with the crystal growth in the supercooled melt. However, for the moment, the lows governing nucleation and crystal growth phenomena for most metals and alloys have not been entirely studied. In this regard, the solidification rate of the melt determines on the basis of the quasi-equilibrium hardening hypothesis [6]. According to this hypothesis, the equilibrium process is observed in case of rather slowly alloy's crystallization (in other words if the diffusional transfer processes by impurity in between liquid and hard phases and intra- phase proceed completely). Then the relative value of the hard phase, which is in equilibrium with the liquid at a temperature $T$, can be determined from the alloy phase diagram by the lever rule.

In the simplest case, the cross section of the hard phase $\psi$ is determined by the piecewise- continuous function, which diagram is shown in Figure 5.

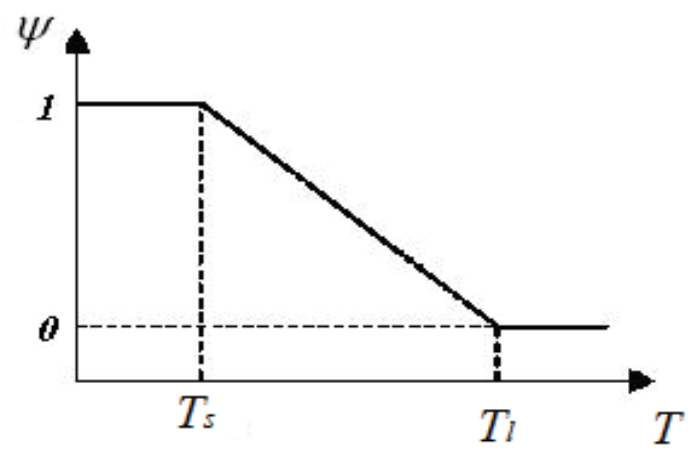

Fig.5 Function of the hard phase content ratio $\psi(T)$ 


$$
\psi(T)=\left\{\begin{array}{l}
0, \text { if } T>T_{l}, \\
\frac{T_{l}-T}{T_{l}-T_{s}}, \text { if } T_{s} \leq T \leq T_{l}, \\
1, \text { if } T<T_{s},
\end{array}\right.
$$

In this case the crystallization rate of the alloy within the interval from $T_{l}$ to $T_{s}$ is a constant value and is equal to:

$$
\frac{\partial \psi}{\partial T}=-\frac{1}{T_{l}-T_{s}}
$$

Taking into account the assumptions of the possible neglect of the heat transfer due to the thermal conductivity in the $\mathrm{Oz}$-direction, we may write the following equation:

$$
\frac{\partial \psi}{\partial \tau}=\frac{\partial \psi}{\partial T} \frac{\partial T}{\partial \tau}
$$

Applying Eq.10 to Eq. 7 , the last can be written as:

$$
\frac{\partial T}{\partial \tau} \rho\left(c-L \frac{\partial \psi}{\partial T}\right)=\frac{\partial}{\partial x}\left(\lambda \frac{\partial T}{\partial x}\right)+\frac{\partial}{\partial y}\left(\lambda \frac{\partial T}{\partial y}\right)
$$

or in greater detail:

$$
\rho \cdot C_{e f} \frac{\partial T}{\partial \tau}=\frac{\partial \lambda}{\partial x} \frac{\partial T}{\partial x}+\lambda \frac{\partial^{2} T}{\partial x^{2}}
$$

where $\tau=0-L_{k} / v$; and $C_{e f}, \lambda, \rho$ - effective heat thermal capacity, conduction and material density of the solidifying ingot.

The effective heat thermal capacity, conduction and material density of solidifying ingot are the piecewise continuous functions [7]:

$$
\begin{aligned}
& C_{e f}(T)=\left\{\begin{array}{l}
C_{l}, \text { if } T>T_{l} ; \\
C_{T}\left(T_{s}\right) \psi+C_{l}\left(T_{l}\right)(1-\psi)-L \frac{\partial \psi}{\partial \tau}, \text { if } T_{s} \leq T \leq T_{l} ; \\
C_{T}(T), \text { if } T<T_{s} ;
\end{array}\right. \\
& \lambda(T)=\left\{\begin{array}{l}
\varepsilon_{\kappa} \lambda_{l}, \text { if } T>T_{l}, \\
\lambda_{\mathrm{T}}+\frac{\varepsilon_{\kappa} \lambda_{l}-\lambda_{\mathrm{T}}}{T_{l}-T_{s}}\left(T-T_{s}\right), \text { if } T_{s} \leq T \leq T_{l}, \\
\lambda_{\mathrm{T}}, \text { if } T<T_{s},
\end{array}\right. \\
& \rho(T)= \begin{cases}\rho_{l}, & \text { if } T>T_{l} ; \\
\rho_{T} \psi+\rho_{l}(1-\psi), \text { if } T_{s}<T<T_{l} ; \\
\rho_{T}(T), \quad \text { if } T<T_{s} .\end{cases}
\end{aligned}
$$

To solve the boundary value problem, the Eq. 11 should be supplemented with the initial and the boundary conditions.

The initial condition defines the temperature field of the melt at the initial time on exit from the melting crucible (if $\tau=0,0<x<b$ )

$$
T(x, 0)=T_{0}=T_{L}+\Delta T_{0} .
$$

For the sides $F G$ and $E H$ of the ingot (Figure 4), which are in contact with the working walls of the mold, the following boundary conditions should be set up.

To determine the boundary conditions for the heattransfer from solidifying liquid was used the method of finite volumes. For the parallelepiped-shaped volume element $f \Delta x$, lying near the cooling wall surface (with an area $f$ ), the heat equilibrium conditions must be met. The capacity of the rejected heat by the mold $q$, at a metal temperature above $T_{s}$, is equal to the phase transition amount of heat (which is be govern by the effective heat capacity of the element of volume $\left.C_{e f} \Delta x \rho \cdot d T / d \tau\right)$ and to amount of heat transferred from the melt volume $-\lambda \delta T / \delta x$.

Accordingly, the boundary conditions are:

for $x=b ; z=\tau \cdot v$,

$$
\begin{aligned}
& \text { if } T>T_{s}, q_{\text {up }}(z)=C_{e f} \cdot \Delta x \cdot \rho \frac{d T}{d \tau}-\left.\lambda \frac{\partial T}{\partial x}\right|_{x=b} ; \\
& \quad \text { if } T \leq T_{s}, q_{\text {up }}(z)=\left.\lambda \frac{\partial T}{\partial x}\right|_{x=b} ;
\end{aligned}
$$

for $x=0 ; z=\tau \cdot v$

$$
\begin{gathered}
\text { if } T>T_{s}, q_{\mathrm{dn}}(z)=C_{e f} \cdot \Delta x \cdot \rho \frac{d T}{d \tau}-\left.\lambda \frac{\partial T}{\partial x}\right|_{x=0} ; \\
\quad \text { if } T \leq T_{s}, \quad q_{\mathrm{dn}}(z)=\left.\lambda \frac{\partial T}{\partial x}\right|_{x=0},
\end{gathered}
$$

where $q_{u p}(z), q_{d n}(z)$, - the heat flow rates towards casting mold interior from top and bottom surfaces of the ingot.

Thus, the obtained Eq. (11) - (17) are the mathematical model of ingot's thermal state in the HCCM.

As a result of this scientific work was developed the mathematical model, which describes the thermal state and the crystallization process of the blank from a precious metal alloy, casting at the small capacity HCCM. Investigations, made on the base of this small capacity HCCM, allowed to determine the behavior in time of the ingot's temperature field at different operating conditions of HCCM

\section{References}

1. V.E. Zinoviev, Heat-transfer properties at high temperatures (Moscow: Ref. ed. Metallurgy, 1989)

2. J. Bast, Horizontal HCC-12000 unit for the continuous casting of semi finished products, Metallurgist, Vol.55, № 1-2. P.116-118 (2011)

3. S.M. Gorbatyuk, I. Yu. Kryukov, A.G. Radyuk, A.E. Titlyanov, The casting mold of horizontal continuous casting machine of ingots from nonferrous metals and alloys, Russian patent № 122052.2012.Bul. №32. 
4. Yu. Bast, Choosing of mold's design by research temperature field in it, Innovative fabricating technology: book of rep. of International scientific and technical conference (M.: Pub.h. MISiS, P. 537-541, 2011)

5. S.M. Gorbatyuk, I. Yu. Kryukov, A.G. Radyuk, A.E. Titlyanov, Water-cooling device of casting mold of horizontal continuous casting machine of ingots from nonferrous metals and alloys, Russian patent №120907.2012.Bul. №28.

6. Yu.A. Samoilovich, Thermal process at continuous steel casting (M.:Metallurgy, 1982)

7. A.I. Tsaplin, Thermophysics in metallurgy, Guide: pub. of Perm State Technical University, 2008)

8. S.M. Gorbatyuk, A.V. Kochanov, Method and equipment for mechanically strengthening the surface of rolling-mill rolls, Metallurgist, 56 (3-4), pp. 279-283 (2012) 